\title{
Gamificación: una innovación en aula para fomentar la motivación
}

\section{Gamification: innovation in the classroom to encourage motivation \\ 1Valentina Calbacho Contreras, ${ }^{2}$ Claudio Díaz Larenas, ${ }^{3}$ Cesar Orsini Sánchez, ${ }^{4}$ Pilar Torres Martínez, \& ${ }^{5}$ Víctor Díaz Narváez}

Calbacho, V., Díaz, C., Orsini, C., Torres, P., \& Díaz, V. (2021). Gamificación: una innovación en aula para fomentar la motivación. Revista Convergencia Educativa, (10), diciembre, 55-64. http://doi.org/10.29035/rce.10.55

[Recibido: 29 octubre, 2021 / Aceptado: 28 noviembre, 2021]

\section{RESUMEN}

Objetivo: Evaluar la aplicación de la gamificación en relación a la satisfacción y la motivación en los estudiantes de Odontología. Método: Estudio piloto evaluativo realizado en estudiantes de tercer año, en la asignatura de Rehabilitación Oral y Preclínico. La clase gamificada constó de 35 alumnos: 25 mujeres y 10 hombres. Los instrumentos utilizados fueron características sociodemográficas, escala motivación situacional, escala de satisfacción académica. Resultados: Al evaluar la satisfacción, los estudiantes evidenciaron resultados positivos con todas las preguntas sobre un 95\% de aprobación. En cuanto a la motivación situacional, las cuatro categorías puntuaron alto, siendo las más altas: motivación intrínseca con un 94 \% y regulación interna un 97\%, ambas en relación con la motivación autónoma. De los 35 estudiantes, 33 puntuaron alto para ambas categorías por lo que el 94\% de la muestra tiene una motivación autónoma. No hubo diferencias significativas por sexo. Conclusión: La gamificación como metodología de la enseñanza y aprendizaje demostró una alta satisfacción y motivación autómona por parte de los estudiantes. Se sugiere realizar nuevos estudios con muestras más significativas para comprobar que la gamificación mejora la satisfacción y la motivación académica favoreciendo un mejor rendimiento académico en educación superior.

Palabras clave: Gamificación, educación, motivación, odontología.

\footnotetext{
${ }_{1}^{1}$ Magíster en Educación Universitaria para Ciencias de la Salud. Universidad del Desarrollo. Concepción, Chile. https://orcid.org/0000-0002-3889-4535 | valentinacalbaho@gmail.com

2 Doctor en Educación. Universidad de Concepción. Concepción, Chile. https://orcid.org/0000-0003-2394-2378 | claudiodiaz@udec.cl

3 Doctor en Educación de Profesiones de la Salud. Universidad de Los Andes. Santiago, Chile. https://orcid.org/0000-0002-5226-3625| cesar.orsini@gmail.com

4 Magíster en Educación Universitaria para Ciencias de la Salud. Universidad San Sebastián. Concepción, Chile. https://orcid.org/0000-0001-8845-8224 | pilar.torres@uss.cl

5 Facultad de Odontología. Universidad Andrés Bello. Santiago, Chile. https://orcid.org/0000-0002-5486-0415 | vicpadina@gmail.com
} 
Calbacho, V., Díaz, C., Orsini, C., Torres, P., \& Díaz, V. (2021). Gamificación: una innovación en aula para fomentar la motivación. Revista Convergencia Educativa, (10), diciembre, 55-64. http://doi.org/10.29035/rce.10.55

\begin{abstract}
Objective: To evaluate the application of gamification concerning satisfaction and motivation in dental students Method: A pilot study was carried out on third year students, in the Oral and Preclinical Rehabilitation subjects. The gamified class consisted of 35 students: 25 women and 10 men. The instruments used were sociodemographic characteristics, situational motivation scale, academic satisfaction scale. Results: When assessing satisfaction, the students showed positive results with all the questions on $95 \%$ approval. In terms of situational motivation, the four categories scored high, the highest being intrinsic motivation with $94 \%$ and Identified regulation $97 \%$, both in relation to autonomous motivation. Of the 35 students, 33 scored high for both categories, so $94 \%$ of the sample had autonomous motivation. There were no significant differences by gender. Conclusions: Gamification as a teaching and learning methodology showed high satisfaction and autonomous motivation on the part of the students. It is suggested to carry out new studies with more significant samples to verify that gamification improves satisfaction and academic motivation, favoring better and academic performance in higher education.
\end{abstract}

Key words: Gamification,Education, Motivation, Dentistry.

\title{
INTRODUCCIÓN
}

La gamificación se entiende como el uso de estrategias, modelos, dinámicas, mecánicas y elementos propios de los juegos en contextos ajenos a éstos, con el fin de enviar un mensaje, un contenido o de cambiar un comportamiento, mediante de una experiencia lúdica motivadora y divertida (Kapp, 2012) siendo una de las estrategias de enseñanza y aprendizaje más innovadora de los últimos años. También se señala que esta estrategia aumentaría la motivación y el esfuerzo de los estudiantes, haciéndoles más partícipes en el proceso de aprendizaje (Cortizo et al., 2011). Por otra parte, la gamificación mejoraría la motivación y la satisfacción, aumentando, incluso, la atención y consolidación de los conocimientos aprendidos (Serna et al., 2016). Aunque existen varios estudios internacionales sobre gamificación en aula, hay muy pocos en relación al área de la salud (Serna et al., 2016, Amer et al., 2011, Beylefeld \& Struwig, 2007, Hannig et al., 2013).

La motivación individual es un factor relevante para la enseñanza-aprendizaje como para generar cambios en la conducta y la gamificación tendría la capacidad de estimular esta motivación (Deterding et al., 2011, Llagostera, 2012, Werbach, 2014, Mauricio et al., 2015). Este cambio promueve que el estudiante pase a tener un papel más activo, autonómo y participativo en la sala de clase (Serna et al., 2016).

Por otra parte, es importante lograr la diversión en los estudiantes pues ésta permite fijar nueva información en el cerebro, de manera que en ella reside la clave del aprendizaje óptimo (Csíkszentmihályi's, 1990); de esta forma, la satisfacción académica que podría generar la gamificación se percibe como el estado placentero que se produce en el estudiante obteniendo un alto nivel de identificación y disfrute en relación a lo que se hace (Salinas et al., 2008). 
En la literatura se puede observar que los estudiantes de Medicina y Odontología presentan mayor nivel de estrés y menor motivación académica (Ahmad et al., 2017, Hawazin et al., 2014, Feldman et al., 2008), y que ésta fluctúa a medida que avanzan en la carrera (Orsini et al., 2016).

Debido a lo expuesto anteriormente, el objetivo de este estudio es evaluar la aplicación de la gamificación como una metodología de la enseñanza y aprendizaje en relación a la satisfacción y la motivación en los estudiantes Odontología.

\section{MATERIALES Y MÉTODOS}

Este estudio piloto evaluativo fue realizado con una población constituida por los estudiantes de tercer año, en el primer semestre de la asignatura de Rehabilitación Oral y Pre-clínico de la Facultad de Odontología de la Universidad del Desarrollo, sede Concepción, Chile, durante el año 2018. Luego de una clase magistral se les hizo una clase gamificada en la Unidad de aprendizaje de Oclusión, en la que participaron 50 estudiantes quedando una muestra de sólo 35 estudiantes: 26 mujeres y 9 hombres. Antes de aplicar la metodología de enseñanza y aprendizaje se les hizo firmar un consentimiento informado que fue aprobado por el comité de ética de la Universidad del Desarrollo, sede Santiago, siguiendo los principios de Helsinki. Los criterios de inclusión fueron estudiantes regulares que asistieran a las actividades teóricas de la asignatura de la Unidad de Oclusión de tercer año de Odontología. Los criterios de exclusión aquellos estudiantes que no firmaron el consentimiento o abandonaron la clase antes de terminar la intervención.

\section{Instrumentos}

1. Características sociodemográficas: estas fueron determinadas por un cuestionario de 10 preguntas de selección múltiple.

2. La Motivación académica: se utilizó escala Motivación Situacional en versión español (Albo et al., 2009). Existen cuatro tipos: la motivación intrínseca, regulación externa, regulación identificada y la amotivación, con subescalas que se relacionan.

3. Satisfacción con la actividad: se realizó la encuesta Satisfacción Académica con el Ambiente Pedagógico modificada por Medrano y validada culturalmente al contexto chileno (Sisto et al., 2008, Melo et al., 2015). 
Calbacho, V., Díaz, C., Orsini, C., Torres, P., \& Díaz, V. (2021). Gamificación: una innovación en aula para fomentar la motivación. Revista Convergencia Educativa, (10), diciembre, 55-64. http://doi.org/10.29035/rce.10.55

\section{Intervención}

La gamificación consistió en un juego presencial utilizando un power point creado por la docente donde la misión era lograr que el paciente cerrara la mandíbula: “Ocluir". Los estudiantes se reunieron en dos grupos para competir pasando un representante de cada grupo adelante según el número asignado. Se establecieron los objetivos de aprendizaje que durante la actividad lúdica debían ser aprendidos por los estudiantes. El primer estudiante del grupo en pasar debía decir stop, mientras la docente pasaba rápidamente las diapositivas, una vez que decía "stop" se abría la pregunta y contestaba. Se presentaron preguntas de alternativas con casillas que tenían distintas consignas lúdicas: bomba, reto, Bonus y medalla de honor con música e imágenes asociadas al power point. Los logros obtenidos fueron establecidos en un tablero de puntuación donde, a través de respuestas correctas al llegar a ciertos puntajes, lograban darles también niveles de estatus: principiante, instructor, maestro, doctor. Por otra parte, existió la posibilidad de retroalimentación desde sus pares como del docente, quien hizo de guía del juego actuando como Avatar. Para el cierre del juego se presentaron los puntajes declarando al grupo vencedor y clarificando dudas puntuales de la materia que pudieron generarse. Por último, en los 10 últimos minutos se aplicaron las encuestas de motivación académica situacional y la satisfacción con la actividad asociada a la gamificación (Tabla 1).

Tabla 1

Cuadro explicativo de las actividades realizadas en el Unidad de aprendizaje de Oclusión según tiempo del módulo académico.

\begin{tabular}{ll} 
Actividad de la Unidad de aprendizaje intervenida & Tiempo \\
\hline Consentimiento y explicación del juego & $10 \mathrm{~min}$ \\
Aplicación ME y sesión Gamificada & $50 \mathrm{~min}$ \\
Cierre de actividad: Dudas y puntaje final & $10 \mathrm{~min}$ \\
Aplicación de encuestas post test M y S & $10 \mathrm{~min}$ \\
\hline
\end{tabular}

M=motivación académica situacional; ME= metodólogia de la enseñanza; S=satisfacción con la actividad. Fuente: Elaborado por los autores.

\section{Análisis estadístico}

Se tabularon los resultados de las encuestas para las características sociodemográficas, motivación académica y satisfacción con la actividad según sexo. Los datos de las variables en estudio fueron examinados, se evaluaron las frecuencias y se presentaron los datos obtenidos en gráficos de barras. Además, se calculó el índice de autodeterminación de Ullrich \& Cox (2009), que considera el siguiente cálculo: 2 x Motivación intrínseca situacional (MIS) + 1 x regulación identificada situacional (RIS)-1 x regulación externa situacional (RES)-2 $\mathrm{x}$ amotivación situacional (AS), siendo todos los datos positivos como sujetos de motivación autónoma y los negativos sujetos de motivación controlada. 


\section{RESULTADOS}

De las características sociodemográficas, vale destacar edad media 20 años (Melo et al., 2015, Ullrich \& Cox, 2009, Caballero et al., 2007, Cabrera \& Galán, 2002, Elliott \& Healy, 2001), sólo un estudiante tenía gratuidad, el 11\% era autofinanciado y el resto tenía beca de arancel y/o beca estatal, el $62 \%$ de la muestra provenía de colegios privados, seis de ellos trabajaban para costear su carrera y sólo el $65 \%$ señaló que Odontología era su carrera de preferencia al postular, donde dos de ellos señalaron que querían cambiarse de carrera.

Al evaluar la satisfacción, los estudiantes evidenciaron resultados positivos en todas las preguntas, siendo las ocho contestadas con sobre un 95\% de aprobación. En esta encuesta, la afirmación: "los profesores son abiertos al diálogo" fue la que obtuvo el más alto porcentaje de la muestra, donde el 98\% señaló estar totalmente de acuerdo. No hubo diferencias significativas por sexo (Figura 1).

\section{Encuesta de satisfacción académica}

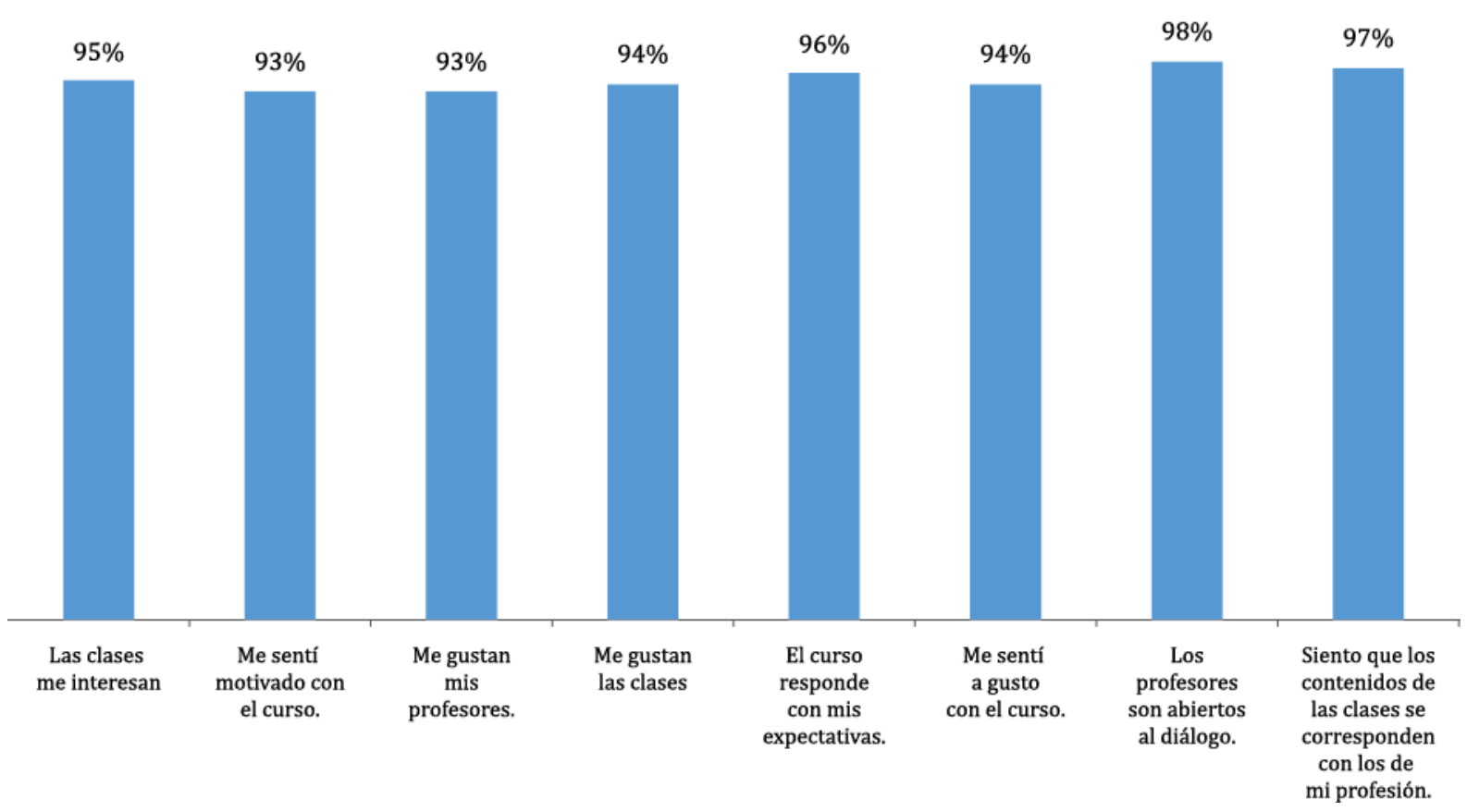

Figura 1. Evaluación de la satisfacción académica en relación a la clase gamificada.

Fuente: Elaborado por los autores.

En cuanto a la motivación situacional, de las cuatro categorías, Amotivación (A), Motivación intrínseca (MI), Regulación externa (RE) y Regulación identificada (RI), las que puntuaron más alto fueron las categorías de Motivación intrínseca, con un 94\% y Regulación interna un 97\%, ambas en relación con la motivación autónoma. De los 35 estudiantes, 33 puntuaron alto para ambas categorías, por lo que el 94\% de la muestra tuvo una motivación autónoma en relación a la gamificación. No hubo diferencias significativas en el sexo (Figura 2). 


\section{Escala de la motivación situacional}

(SIMS)

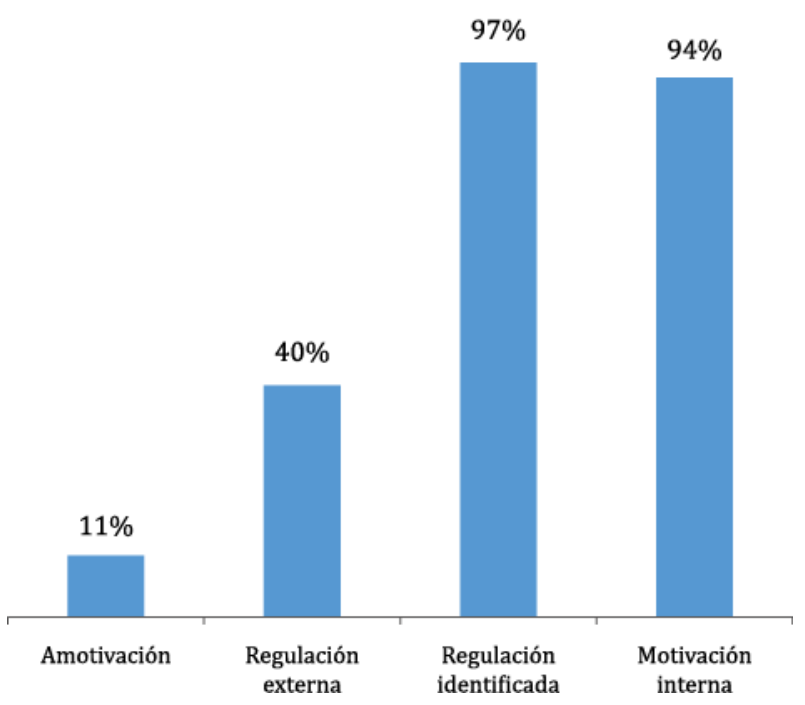

Figura 2. Categorías de motivación situacional y porcentaje obtenido posterior a la gamificación.

Fuente: Elaborado por los autores.

Al calcular el índice de autodeterminación, se observó que hubo 30 estudiantes con valores positivos, es decir, 86\% de la muestra presentó motivación autónoma y sólo cinco estudiantes presentaron motivación controlada, constituyendo el 14\% de la muestra.

\section{DISCUSIÓN}

El Espacio Europeo de Educación Superior se fundamenta en el aprendizaje autónomo de competencias por parte del estudiante universitario (Martínez, 2011). La gamificación demostró una alta satisfacción y motivación autómona por parte de los estudiantes. Esto coincide con otros trabajos realizados con respecto a la satisfacción en el uso de juegos serios, donde los estudiantes estaban satisfechos con el juego de vinculación de dentina pues disfrutaron la actividad y creyeron que podría ayudarlos a mantener el interés en el tema (Amer et al., 2011). Del mismo modo, en otro estudio (Hannig et al., 2013) informaron una alta calificación para la usabilidad del juego a partir del cuestionario de comentarios, donde informaron resultados positivos en motivación, atmósfera de aprendizaje adecuada, humor, retroalimentación y aprendizaje incidental.

En Chile la satisfacción académica es un tema escasamente abordado y, menos aún, en relación a una metodología de aprendizaje y enseñanza como la gamificación, por lo cual el presente trabajo constituye un precedente para impulsar investigación en torno a este tema, particularmente porque la satisfacción académica como la motivación han demostrado ser un constructo de gran importancia para comprender el comportamiento de los estudiantes (Amer et al., 2011) y, según muestra la literatura, se relaciona con la retención, el rendimiento académico, la autoeficacia académica, la satisfacción general con la vida y el buen 
funcionamiento académico(Caballero et al., 2007, Cabrera \& Galán, 2002, Elliott \& Healy, 2001, Fernández et al., 2007). Por otra parte, la importancia de implementación de este piloto radica en evidenciar que la gamificación como metodología de la enseñanza requiere de un trabajo previo importante en cuanto a la confección del juego, que debe cumplir con los elementos necesarios en cuanto a su dinámica y narrativa, donde los estudiantes deben involucrarse y tomar sus propias decisiones participando en un entorno social que los reconoce con retroalimentación constante por parte del tutor. Así mismo la incorporación de los aspectos clave como: diversión, motivación, autonomía, progresividad, retroalimentación inmediata y tratamiento del error, son varios aspectos a considerar en el diseño la actividad gamificada (Llorens et al., 2016).

Se sugiere realizar nuevos estudios con muestras más significativas para comprobar cómo la gamificación mejora la satisfacción y la motivación académica en relación al rendimiento académico en educación superior.

Este estudio tiene ciertas limitaciones, tales como la mayor dificultad en hacer participar a estudiantes más tímidos, preguntas de igual dificultad, ajustar los tiempos ya que algunos se ponían nerviosos demorando más en contestar. Además, a veces las respuestas no estaban completas o no estaban 100\% correctas por lo que hay que manejar preguntas dicotómicas y bien dirigidas en el juego.

Conflicto de interés: Los autores declaran no tener ningún conflicto de interés.

\section{REFERENCIAS BIBLIOGRÁFICAS}

Ahmad, F. A., Karimi, A. A., Alboloushi, N. A., AL-Omari, Q. D., AlSairafi, F. J., \& Qudeimat, M. A. (2017). Stress Level of Dental and Medical Students: Comparison of Effects of a Subject-Based Curriculum versus a Case-Based Integrated Curriculum. Journal of Dental Education, 81(5), 534-544. https://doi.org/10.21815/JDE.016.026

Albo, M., Núñez, J., Gregorio, J., \& Grijalvo, F. (2009). Un modelo explicativo del bienestar Psicológico en la Universidad. Revista Mexicana de investigación en Psicología, 26(1), 41-50. https://www.redalyc.org/pdf/2430/243016317004.pdf

Amer, R. S., Denehy, G. E., Cobb, D. S., Dawson, D. V., Cunningham-Ford, M. A., \& Bergeron, C. (2011). Development and evaluation of an interactive dental video game to teach dentin bonding. Journal of Dental Education, 75(6), 823-831. https://doi.org/10.1002/j.0022-0337.2011.75.6.tb05111.x

Beylefeld, A., \& Struwig, M. (2007). Gaming approach to learning medical microbiology: Students'

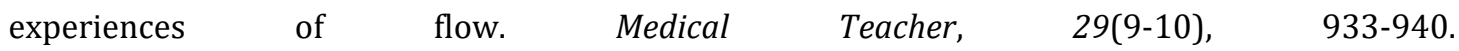
https://doi.org/10.1080/01421590701601550 
Caballero, C., Abello, R., \& Palacio, J. (2007). Relación del burnout y el rendimiento académico con la satisfacción frente a los estudios en estudiantes universitarios. Avances en Psicología Latinoamericana, 25(2),

98-111. https://revistas.urosario.edu.co/index.php/apl/article/view/1208/1076

Cabrera-Guillén, P., \& Galán Delgado, E. (2002). Factores contextuales y rendimiento académico. Revista electrónica interuniversitaria de formación del profesorado, 5(3), 4. https://dialnet.unirioja.es/servlet/articulo?codigo=1034506

Cortizo, J., Carrero. F., Monsalve, B., Velasco, A., Díaz del Dedo, L., \& Pérez, J. (2011). Gamificación y Docencia: Lo que la Universidad tiene que aprender de los Videojuegos [Sesión de conferencia]. Retos y oportunidades del desarrollo de los nuevos títulos en educación superior VIII Jornadas Internacionales de Innovación Universitaria, Madrid, España. https://abacus.universidadeuropea.es/bitstream/handle/11268/1750/46_Gamificacion.pdf

Csíkszentmihályi's, M. (1990). Flow: The psychology of optimal experience. Harper \& Row. https://www.researchgate.net/publication/224927532_Flow_The_Psychology_of_Optimal_Exper ience

Deterding, S., Dixon, D., Khaled, R., \& Nacke, L. (2011). From game design elements to gamefulness: defining" gamification". In Proceedings of the 15th international academic MindTrek conference: Envisioning future media environments (pp. 9-15). https://doi.org/10.1145/2181037.2181040

Elliott, K., \& Healy, M. (2001). Key factors influencing student satisfaction related to recruitment and retention. Journal of Marketing for Higher Education, 10(4), 1-11. https://doi.org/10.1300/J050v10n04_01

Feldman, L., Goncalves, L., Chacón, G., Zaragoza, J., Bages, N., \& De Pablo, J. (2008). Relaciones entre estrés académico, apoyo social, salud mental y rendimiento académico en estudiantes universitarios $\begin{array}{llll}\text { venezolanos. } & \text { Universitas } & \text { 739-751. }\end{array}$ http://www.scielo.org.co/scielo.php?script=sci_arttext\&pid=S1657-92672008000300011

Fernández, E., Fernández, S., Álvarez, A., \& Martínez, P. (2007). Éxito académico y satisfacción de estudiantes con la enseñanza universitaria. Revista Electrónica de Investigación y Evaluación Educativa, 13(2), 203-214. https://doi.org/10.7203/relieve.13.2.4207

Hannig, A., Lemos, M., Spreckelsen, C., Ohnesorge-Radtke, U., \& Rafai, N. (2013). Skills-o-mat: computer supported interactive motion- and game- based training in mixing alginate in dental education. Journal of Educational Computing Research, 48(3), 315-343. https://doi.org/10.2190/EC.48.3.c 
Hawazin, EW., Allison, PJ., Kumar, R., Mancini, L., Lambrou, A., \& Bedos, C. (2014). A Systematic Review of Stress in Dental Students. Journal of Dental Education, 78(2), 226-242. https://assetpdf.scinapse.io/prod/1835919563/1835919563.pdf

Kapp, M. (2012). The gamification of learning and instruction: Game-based methods and strategies for training and education. Pfeiffer.

Llagostera, E. (2012). On Gamification and Persuasion [Sesión de conferencia]. XI SBC-Proceeding of SB Games, Brasilia, Brasil. http://www.sbgames.org/sbgames2012/proceedings/papers/gamesforchange/g4c-02.pdf

Llorens-Largo, F., Gallego-Durán, F., Villagrá-Arnedo, C., Compañ, P., Satorre-Cuerda, R., \& Molina-Carmona, R. (2016). Gamificación del proceso de aprendizaje: lecciones aprendidas. VAEP-RITA, 4(1), 25-32. http://rita.det.uvigo.es/VAEPRITA/201603/uploads/VAEP-RITA.2016.V4.N1.A4.pdf

Martínez, J. (2011). La motivación para aprender en el espacio europeo de educación superior. Cuadernos de Educación y Desarrollo, Cuadernos de Educación y Desarrollo, (25). https://ideas.repec.org/a/erv/cedced/y2011i2549.html

Mauricio, M., Serna, E., \& Vallés, S. (2015). Experiencias en la aplicación de la gamificación en 1o Curso de Grado de Ciencias de la Salud. En In-Red 2015-Congreso Nacional de Innovación Educativa y de Docencia en Red. Universitat Politècnica de València. http://dx.doi.org/10.4995/INRED2015.2015.1583

Melo, B., Moreira, L. O., Villalobos, C. P., Araneda, G. T., Calvo, P. M., Kother, A. M., Matus, O., Bastías, N., \& Bustamante, C. (2015). Estructura Factorial y Confiabilidad del Cuestionario de Satisfacción Académica en Estudiantes de Medicina Chilenos. Revista Iberoamericana de Diagnóstico y Evaluación, 2(40), 73-82. https://www.redalyc.org/pdf/4596/459645432008.pdf

Orsini, C., Binnie, VI., \& Wilson, S. L. (2016). Determinants and outcomes of motivation in health professions education: A systematic review based on Self- determination theory. Journal of Educational Evaluation for Health Professions, 13, 19. http://dx.doi.org/10.3352/jeehp.2016.13.19

Salinas, A., Morales, J., \& Martinez, P. (2008). Satisfacción del estudiante y calidad universitaria: un análisis explicatorio en la unidad académica multidisciplinaria agronomía y ciencias de la Universidad Autónoma de Tamaulipas, México. Revista de Enseñanza Universitaria, 31, 39-55. https://dialnet.unirioja.es/servlet/articulo?codigo=3900906

Serna, E., Mauricio, M., San Miguel, T., \& Megías, J. (2016). Experiencia de gamificación en Docencia Universitaria: aprendizaje activo y entretenido. En In-Red 2016. II Congreso nacional de innovación educativa $y$ docencia en red. Universitat Politècnica de València. https://doi.org/10.4995/INRED2016.2016.4292 
Calbacho, V., Díaz, C., Orsini, C., Torres, P., \& Díaz, V. (2021). Gamificación: una innovación en aula para fomentar la motivación. Revista Convergencia Educativa, (10), diciembre, 55-64. http://doi.org/10.29035/rce.10.55

Sisto, F. F., Muniz, M., Bartholomeu, D., Pasetto, N. S. V., de Oliveira, A. F., \& Lopes, W. M. G. (2008). Estudo para a Construção de uma Escala de Satisfação Acadêmica para Universitários. Avaliação Psicológica, 7(1), 45-55. https://www.redalyc.org/pdf/3350/335027183007.pdf

Ullrich-French, S., \& Cox, A. (2009). Using Cluster Analysis to Examine the Combinations of Motivation Regulations of Physical Education Students. Journal of Sport and Exercise Psychology, 31(3), 358379. https://doi.org/10.1123/jsep.31.3.358

Werbach, K. (2014). (Re)Defining Gamification: A Process Approach. En A. Spagnolli, L. Chittaro, \& L. Gamberini (Eds.) Persuasive Technology. PERSUASIVE. Lecture Notes in Computer Science, 8462, 266-272. Springer. https://doi.org/10.1007/978-3-319-07127-5_23

\section{Datos de correspondencia}

Valentina Calbacho

Magíster en Educación Universitaria para Ciencias de la Salud.

Universidad del Desarrollo

Concepción, Chile.

Dirección postal: 4030000

ORCID ID: https://orcid.org/0000-0002-3889-4535

Email:valentinacalbaho@gmail.com 